\section{Herausforderungen transdisziplinären Arbeitens im Reallabor „Wissensdialog Nordschwarzwald"}

\author{
von Regina Rhodius, Michael Pregernig und \\ Barbara Koch, Universität Freiburg
}

Das Reallabor „Wissensdialog Nordschwarzwald" ist eng an den Nationalpark Schwarzwald gekoppelt: Wie lassen sich Chancen nutzen, die der Nationalpark für die nachhaltige Entwicklung der Region bietet? Was lässt sich aus den im Nationalparkgebiet stattfindenden ökologischen Prozessen lernen? Dies sind die zentralen Forschungsfragen des Projekts. Der vorliegende Beitrag stellt die Herausforderungen des spezifischen transdisziplinären Designs des Reallabors in den Vordergrund. Basierend auf Interviews mit Projektbeteiligten, dem Austausch in Projektgremien und eigenen Beobachtungen reflektieren die Autorlnnen zentrale Designelemente sowie Kontextbedingungen des Projektes. Angesichts der besonderen Gegebenheiten des ländlichen Raumes postulieren sie den Bedarf, eine stärker an diesen angepasste Reallaborforschung zu entwickeln.

The Real-world Laboratory "Knowledge Dialogue Northern Black Forest" is closely linked to the Black Forest National Park in Baden-Württemberg, Germany: How can one actually take advantage of the opportunities a national park offers for regional transformation towards sustainability, and what can be learnt from ecological processes in the park area? These are the project's core questions. The following article focuses on the transdisciplinary project design and its challenges. Based on interviews with project members, discussions within project committees and own observations, the authors reflect on essential design elements and context conditions of the project. On this basis, they postulate a need for better adapting the real-world laboratory research to the specific conditions of rural areas.
1 Reallaborforschung im ländlichen Raum im Kontext eines sich etablierenden Nationalparks

Nachhaltige Entwicklung ist eine gesellschaftliche Aufgabe, bei der ländlichen Räumen eine Schlüsselrolle zukommt (SRU 1996; Friedel/ Spindler 2009; Marsden 2009): Diese stellen den größten Teil der natürlichen Ressourcen bereit; sind wesentlicher Träger der Energiewende; sie beinhalten zugleich Hotspots der Biodiversität und sind zentral für die Erholung der Bevölkerung. In sozioökonomischer Hinsicht unterliegen ländliche Räume spezifischen langfristigen Transformationsprozessen, so z. B. in Land- und Forstwirtschaft oder im Tourismus. In soziokultureller Hinsicht sind sie schließlich durch Wissenskulturen geprägt, die im Kontrast zu urbanen Erfahrungswelten stehen können (Bruckmeier/ Tovey 2009; Reimer/Brett 2013; Shortall 2013). Als einziges der bisher 14 vom baden-württembergischen Ministerium für Wissenschaft, Forschung und Kunst (MWK) geförderten Reallabore bewegt sich das Projekt „Wissensdialog Nordschwarzwald" (WiNo) in einem ländlichen Kontext (https://mwk.baden-wuerttemberg.de/ de/forschung/forschungspolitik/wissenschaftfuer-nachhaltigkeit/reallabore/).

Das Projekt WiNo ist eng an den im Januar 2014 neu gegründeten Nationalpark Schwarzwald gekoppelt (Abb. 1). Mit dem Gründungsprozess einher gingen intensive politische und wissenschaftliche Debatten (Eser et al. 2013; Hubo/Krott 2015). Im Zentrum stand zunächst die (ökologische) Entwicklung des Schutzgebietes und der angrenzenden Bereiche. Gleichzeitig entwickelte sich eine breitere sozioökonomische Diskussion, die die Auswirkung des Parks auf die soziale und wirtschaftliche Entwicklung der ganzen Region in den Fokus nahm. Vor dem Hintergrund dieser Debatte ist es Ziel von WiNo, die Entwicklung des Nationalparks Schwarzwald forschend $\mathrm{zu}$ begleiten. Wie lassen sich Chancen nutzen, die der Nationalpark für die nachhaltige Entwicklung der Region bietet? Und was lässt sich aus den im Nationalparkgebiet stattfindenden ökologischen Prozessen lernen? Diese beiden Kernfragen untersucht das Projekt WiNo, das von einem interdisziplinären Team der Albert-Ludwigs-Universität Freiburg, der 


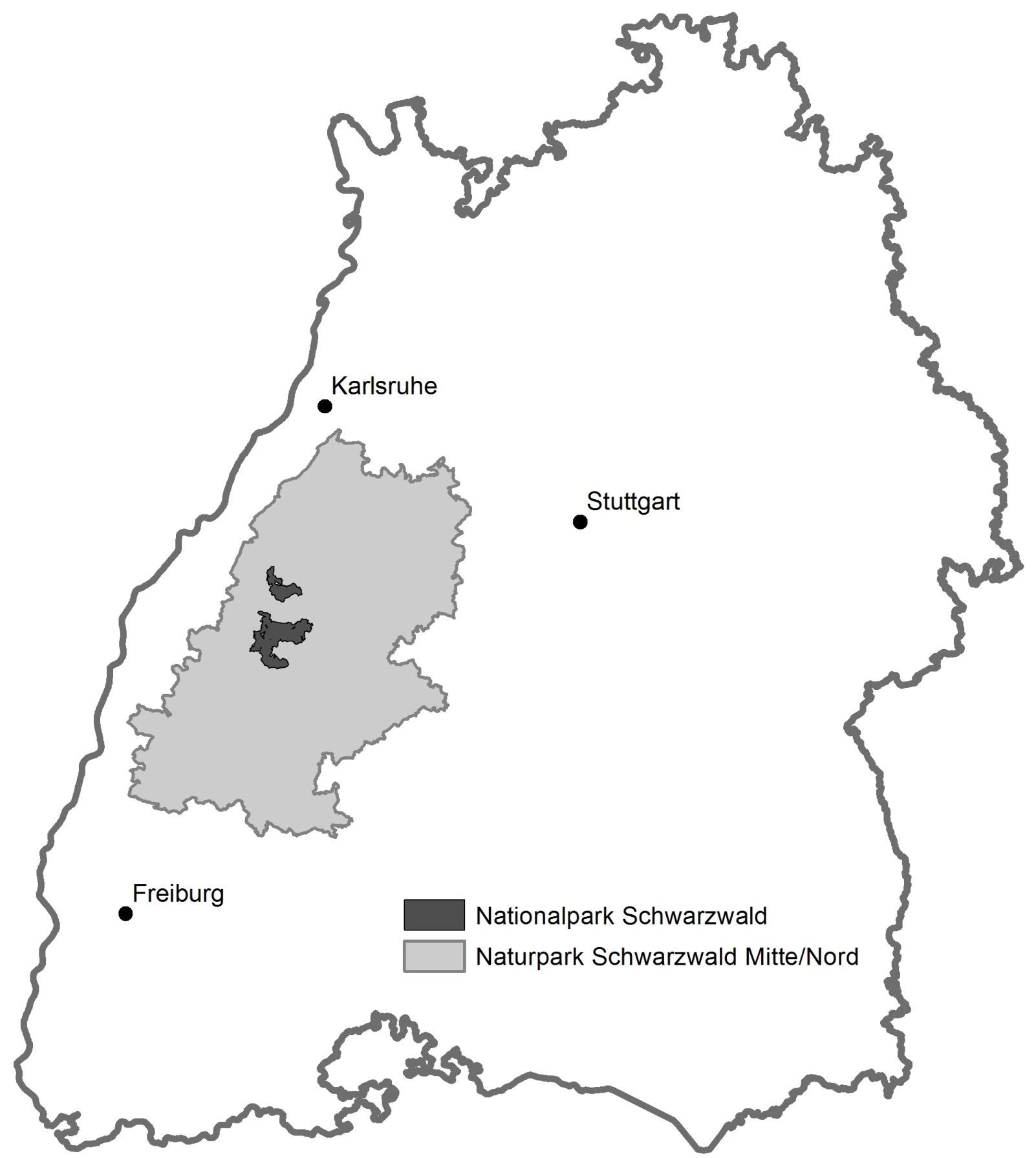

WiNo bezieht sich nicht allein auf das Nationalparkgebiet, sondern betrachtet die Interaktionen zwischen dem Nationalpark und der Region. In einem weiteren Sinn gefasst, entspricht das Projektgebiet daher in etwa den Grenzen des Naturparks Schwarzwald Mitte/Nord.

Quelle: Eigene Darstellung auf Basis der Karte der Landesanstalt für Umwelt, Messungen und Naturschutz Baden-Württemberg und OpenStreetMap 
Hochschule für Forstwirtschaft Rottenburg, des Öko-Instituts e.V., der Forstlichen Versuchs- und Forschungsanstalt Baden-Württemberg sowie der EVOCO GmbH getragen wird. Die Verwaltungen des Nationalparks Schwarzwald und des Naturparks Schwarzwald Mitte/Nord wirken als Praxispartner mit.

\section{Die theoretische Verortung des transdisziplinären Projektdesigns}

Das Projekt WiNo orientiert sich konsequent an Prinzipien der Transdisziplinarität; das Projektdesign lehnt sich dabei an das dreistufige Prozessmodell von Jahn et al. (2012) an. Die erste Phase dieses Modells wird in der jüngeren Literatur zu Nachhaltigkeitstransformationen oft unter dem Begriff des „Co-Designs“ gefasst. Co-Design steht dabei einmal für die partizipative Entwicklung und Implementation von Forschungsprogrammen (z. B. Future Earth 2013). Co-Design hat zudem Eingang in die prozedurale Ausgestaltung von Forschungsprojekten gefunden (van Kerkhoff 2014) und findet sich schließlich als Leitprinzip der Gestaltung von - bislang vor allem städtischen - Initiativen des Transition-Managements (Nevens et al. 2013).

Die zweite Phase eines transdisziplinären Projekts wird häufig unter dem Begriff der „COProduktion" gefasst. Auch dieser Begriff wird in verschiedenen Kontexten unterschiedlich verstanden: in der Wissenschaftsforschung als streng analytisches Konzept, das sichtbar macht, wie sich Prozesse der Schaffung und Stabilisierung von wissenschaftlicher und gesellschaftlicher Ordnung bedingen (Jasanoff 2004); im Feld der „Science-Policy Studies“ als Instrument, um Prozesse der wissenschaftlichen Politikberatung effektiver gestalten zu können (Hegger et al. 2012; Bauer et al. 2016), und im Kontext transdisziplinärer Forschungsprojekte wie WiNo als Ausdruck der Einbeziehung nicht-wissenschaftlicher Akteure in die wissenschaftliche Erkenntnisproduktion (Pohl et al. 2010; Polk 2015).

Die dritte Phase kann, wie kürzlich von einer Arbeitsgruppe im Deutschen Komitee für Nachhaltigkeitsforschung in Future Earth vorgeschlagen, mit dem Begriff der „Co-Dissemination " gefasst werden. Alternativ finden sich in der Literatur die Begriffe der „In-Wert-Setzung“ (Matthiesen/Bürkner 2004) oder der ,transdisziplinären Integration“ (Bergmann/Jahn 2005). Gerade das letztgenannte Konzept verweist nachdrücklich darauf, dass von transdisziplinären Projekten sowohl Impulse für Diskurse und Innovationen im Praxisfeld als auch in der Wissenschaft ausgehen können bzw. sollten.

\section{Phasen, Instrumente und Strukturen des transdisziplinären Projektdesigns}

In Anlehnung an die o. g. Literatur ist das Projekt WiNo in die drei Phasen Co-Design (1), Co-Produktion (2) und Co-Dissemination (3) unterteilt. Diese werden im Folgenden beschrieben und im nächsten Abschnitt einer ersten, vorläufigen Bewertung unterzogen.

1. Co-Design (Januar - September 2015): Folgende Schritte dienten der Einbindung regionaler Akteure in die Entwicklung des Forschungsprogramms:

a) Befragung regionaler AkteurInnen: Bereits in der Phase der Antragstellung benannten regionale Akteure aus Politik, Wirtschaft und Gesellschaft wichtige Themen rund um die nachhaltige Entwicklung des Nationalparks und seines Umfelds. Basierend auf den Umfrageergebnissen wurden sechs prioritäre Themenfelder identifiziert und wissenschaftliche Teams gebildet.

b) Entwicklung von Forschungsideen: Mit dem Projektstart im Januar 2015 entwickelten die WissenschaftlerInnen die sechs Themenfelder aus der Phase der Antragstellung zu Forschungsideen. Dies erfolgte in Abstimmung mit den Verwaltungen des Nationalpark (NLP) Schwarzwald, des Naturpark Schwarzwald Mitte-Nord und in Gesprächen mit VertreterInnen von Tourismus und regionaler Wirtschaft.

c) Vorbereitung und Durchführung der Wissensmesse: Die Wissensmesse bildete das Kernelement des Co-Designs. Ihr Ziel war es, gemeinsam mit AkteurInnen und BürgerInnen konkrete Forschungsfragen zu entwickeln, die sowohl für die Wissen- 
schaft, als auch die Region hohe Relevanz aufweisen. Unter dem Motto „Wissensdialog Nordschwarzwald - fragen, diskutieren, forschen" luden die Projektpartner am 20. Juni 2015 in das K(ult)urhaus Bad Peterstal ein. Über 70 Personen aus der Region - vorwiegend VertreterInnen regionaler Institutionen und Interessengruppen - folgten der Einladung und diskutierten die Forschungsideen.

d) Ausarbeitung von Projektskizzen: Im Nachgang der Wissensmesse überarbeiteten die Forschungsteams ihre Projektideen und reichten ihre Projektskizzen bei einem aus externen Wissenschaftlern bestehenden Kuratorium ein.

e) Festlegung des Forschungsprogramms: Auf Basis der Kuratoriumsempfehlungen wählte das projektinterne Steuerungsboard im September 2015 die Forschungsprojekte aus und legte die Verteilung der Finanzmittel fest. Die ausgewählten Projekte decken ein breites Themenspektrum ab: Nachhaltige digitale Mobilitätskonzepte und innovative Produkte des Gesundheitstourismus, die historische Entwicklung der Kulturlandschaft, die Ausbreitung von Borkenkäfern sowie Dynamiken der natürlichen Waldentwicklung stehen ebenso auf der Agenda wie Instrumente zur Wegeplanung im Nationalpark, die Wahrnehmung von Wildtieren durch AnwohnerInnen und BesucherInnen der Region und das lokale Wissen der Bevölkerung zum Nationalpark.

2. Co-Produktion (Oktober 2015-April 2017):

Die im Co-Design entwickelten Projekte werden derzeit in der bis April 2017 laufenden Forschungsphase wissenschaftlich bearbeitet. Hierzu beziehen die Forschungsprojekte die verschiedenen regionalen Akteure je nach Thema in recht unterschiedlicher Intensität ein. Das Spektrum reicht dabei von regelmäßigen Abstimmungen mit Praxispartnern über die Befragung von AnwohnerInnen und BesucherInnen bis zur Durchführung von Expertenworkshops. Die geplante Einbeziehung interessierter BürgerInnen in das Monitoring von Versuchsflächen (Citizen Science) erwies sich aufgrund der nun definierten Schutzbestimmungen des Nationalparks als nicht realisierbar: In der Kernzone des Nationalparks sollen menschliche Einflüsse weitestgehend vermieden werden; Citizen Science-Aktivitäten sind mit diesem Ziel kaum vereinbar.

Transdisziplinarität wird in der Forschungsphase auch durch spezielle finanzielle Instrumente unterstützt: Durch ein TandemProgramm können WissenschaftlerInnen und PraktikerInnen die Arbeit der ,anderen Seite“ kennenlernen; Mobilitätsstipendien sollen es WissenschaftlerInnen und AkteurInnen aus der Region erlauben, Erfahrungen mit anderen Nationalparks im In- und Ausland auszutauschen; die Finanzierung von forschenden Lehr- und Lernprojekten ermöglicht die Bearbeitung von Projektthemen durch Studierende. Prinzipien der Inter- und Transdisziplinarität werden auch durch strukturelle Maßnahmen, wie eine gemeinsame Geschäftsstelle, befördert. Das halbjährlich stattfindende Statuskolloquium wird durch eine die verschiedenen Forschungsprojekte verbindende „Querschnitts-AG“ ergänzt. Der Verankerung in der Region dient die Veranstaltungsreihe „Wissensdialog vor Ort“.

3. Co-Dissemination (Mai - Dezember 2017): Ziel der dritten Projektphase, die unter dem Titel „SynPuls“ steht, ist einmal die Zusammenführung der wissenschaftlichen Ergebnisse (,Syn“) und darauf aufbauend das Setzen von Impulsen für Problemlösungen und zukünftige Projekte in der Region („Puls“). Die Ergebnisse werden dabei zunächst in Wissenschaft-Praxis-Tandems aufbereitet und diskutiert. Darauf aufbauende SynPulsVeranstaltungen in der Region sollen interessierte BürgerInnen und regionale AkteurInnen mit den Wissenschaftsteams und PraxispartnerInnen in Austausch bringen.

Das in WiNo gewählte transdisziplinäre Design beinhaltet mit den oben geschilderten Formaten zur Einbindung von AkteurInnen einen typischen Wechsel zwischen Phasen eines breit angelegten, die interessierte Bevölkerung ansprechenden Austauschs, einer engen Zusammenarbeit zwischen Wissenschaft und Praxis in überschaubaren, teilweise bilateralen Formaten und der rein 
wissenschaftlichen Auseinandersetzung mit den Forschungsthemen (vgl. Hurni/Wiesmann 2004).

\section{Von Designentscheidungen und Kontextbedingungen - Fünf Reflexionen}

Die folgenden fünf Reflexionen beziehen sich sowohl auf zentrale, das transdisziplinäre Projektdesign betreffende Entscheidungen, als auch auf die spezifischen Kontextbedingungen von WiNo und speisen sich aus unterschiedlichen Quellen. Sie basieren zum einen auf Interviews, die im Rahmen einer studentischen Qualifikationsarbeit geführt wurden und im Zuge derer 13 von insgesamt rund 20 WiNo-WissenschaftlerInnen zu ihren Erfahrungen mit dem transdisziplinären Design befragt wurden. Die ausgewählten InterviewpartnerInnen repräsentierten dabei das inhaltliche Spektrum und die verschiedenen Gremien des Projektes. Zum anderen gehen sie auf den Austausch in den Projektgremien und die Selbstreflexion der im Projekt tätigen AutorInnen zurück. Die Reflexionen beziehen sich auf die bislang gewonnenen Erfahrungen hinsichtlich 1) Form und Intensität des Austauschs zwischen Wissenschaft und Gesellschaft, 2) der Iterativität des Co-Design-Prozesses, und 3) des kompetitiven Ansatzes der Themenauswahl. Anknüpfend an diese drei Aspekte des internen Designs werden 4) die Einbettung des Projekts in ein „politisiertes" Umfeld, und 5) Charakteristika eines Reallabors im ländlichen Raum reflektiert.

1. Form und Intensität des Austauschs zwischen Wissenschaft und Gesellschaft: Den zentralen Baustein des WiNo-Co-Designs bildete die Wissensmesse, bei der die Forschungsfragen im Austausch zwischen Wissenschaft, Praxis und Gesellschaft entwickelt werden sollten. Die Frage, inwieweit dies tatsächlich gelungen ist, wird von den beteiligten WissenschaftlerInnen sehr unterschiedlich beantwortet. So wird z. B. die Tatsache, dass die etwa 70 teilnehmenden Personen vorwiegend VertreterInnen regionaler Institutionen und Interessengruppen darstellten und die über Gemeindeblätter und Pressearbeit ebenfalls angesprochene nicht-organisierte
Bürgerschaft kaum vertreten war, verschieden interpretiert.

Ein Teil der WissenschaftlerInnen ist der Meinung, dass die richtigen Personen und Gruppen erreicht wurden. Einzelne Befragte vertreten dabei die Ansicht, dass BürgerInnen ohnehin mit der Erarbeitung spezifischer Forschungsfragen überfordert gewesen wären. Dass vorwiegend regionale AkteurInnen, die mit der Thematik vertraut waren, anwesend waren, hätte produktive Diskussionen erst ermöglicht. Demgegenüber fällt das Urteil anderer WissenschaftlerInnen deutlich kritischer aus: Sie hätten sich ein größeres $\mathrm{Maß}$ an Interaktion mit BürgerInnen erwartet und sehen dementsprechend das Ziel der Wissensmesse, eine breite Öffentlichkeit zu erreichen, als verfehlt an.

Nicht nur die Frage, wer mit der Veranstaltung erreicht wurde, auch die Art der Interaktion wird unterschiedlich beurteilt: Für die einen bot die Wissensmesse eine effektive Plattform zur Öffnung der Forschungsagenden; für die anderen agierte die Wissenschaft zu selbstbezogen und hätte den BürgerInnen zu wenig zugehört. Die divergierenden Einschätzungen machen deutlich, dass „Beteiligung" im Kontext transdisziplinärer Projekte mit sehr unterschiedlichen Wahrnehmungen und Erwartungen der beteiligten WissenschaftlerInnen verbunden ist; auch deren Bereitschaft, sich auf die Mitarbeit von NichtWissenschaftlerInnen einzulassen, scheint recht unterschiedlich ausgeprägt zu sein. Eine projektinterne Verständigung auf Kriterien für eine erfolgreiche Einbeziehung von nichtwissenschaftlichen AkteurInnen könnte hier für eine größere Kongruenz sorgen.

2. Iterativität des Co-Design-Prozesses: Das zu Projektbeginn geplante Vorgehen erfuhr im Laufe des Prozesses schrittweise Anpassungen, die dazu führten, dass sich die Co-Design-Phase von geplanten vier auf letztlich neun Monate verlängerte. Einige befragte WissenschaftlerInnen sahen diesen Prozess als notwendig an, um tatsächlich zu neuen Forschungsideen zu gelangen. Andere empfanden die Phase des Co-Designs als Ablenkung von der eigentlichen Forschung 
und die Änderungen im Prozessverlauf als einen unfairen Wechsel von Spielregeln. Die Äußerungen lassen ein Spannungsfeld zwischen der Flexibilität, die transdisziplinäres Arbeiten erfordert, und dem Wunsch der Beteiligten nach vorhersehbaren Verfahrensschritten erkennen.

3. Kompetitiver Ansatzes der Themenauswahl: Auch der Wettbewerbscharakter wurde unterschiedlich beurteilt: Von einigen der WissenschaftlerInnen wurde dieser als hilfreicher Zwang betrachtet, um tatsächlich neue Ideen in die eigene Forschung aufzunehmen und mit Methoden der Akteursbeteiligung zu experimentieren. Andere äußerten, man habe über neun Monate intensiv gearbeitet, ohne zu wissen, ob das angestrebte Projekt letztlich auch finanziert würde. Damit wird ein Spannungsfeld zwischen dem Wunsch nach die Offenheit fördernden Anreizstrukturen und dem Bedürfnis nach Planungssicherheit sichtbar, der auch durch eine geschickte und transparente Prozessgestaltung nur bedingt auflösbar ist.

4. Einbettung des Projekts in ein ,politisiertes" Umfeld: Während sich viele Nachhaltigkeitsforschungsprojekte tendenziell eher konsensorientierten Themen widmen (Bauer/Pregernig 2013; Penker/Muhar 2015), ist WiNo in einem vergleichsweise ,politisierten" Umfeld angesiedelt. Dies birgt sowohl Chancen als auch Risiken in sich. So haben die vielen abgeschlossenen und laufenden Beteiligungsprozesse auf Seiten der Bevölkerung und der Interessensvertretungen zu einer gewissen „Partizipationsmüdigkeit“ in der Region geführt. Die enge Zusammenarbeit mit der im Aufbau befindlichen Nationalparkverwaltung bietet die Möglichkeit, Themen $\mathrm{zu}$ setzen und gegebenenfalls zu verstetigen. Andererseits erfordern die große inhaltliche Nähe von Verwaltung und Projekt und die politische Brisanz einzelner Themen einen für Forschungsprojekte außergewöhnlich hohen Abstimmungsbedarf. Damit erfordert die Arbeit in WiNo einen delikaten Balanceakt, um zwar als aktiver Impulsgeber in der Region, nicht jedoch als Störfaktor in einer politisch sensitiven Arena wahrgenommen zu werden.
5. Charakteristika eines Reallabors im ländlichen Raum: Infolge der Verortung von WiNo in einem ländlichen Kontext sind die einzelnen Forschungsprojekte in WiNo auf eine große Fläche verteilt. Der damit verbundene hohe Grad an Dezentralität macht es schwierig, in der gesamten Region Präsenz zu zeigen. Der ländliche Raum weist schließlich auch soziokulturelle Spezifika auf. Dies sei an einem Beispiel festgemacht: Bereits zu Projektbeginn wurde der Begriff „Reallabor“ von Praxispartnern sehr kritisch hinterfragt. Das „Labor“ war für sie weniger mit der Freiheit „sozialer Experimentierräume“, als vielmehr mit der Vorstellung eines klinischexperimentellen Settings verbunden - bis hin zur Assoziation, die Einheimischen würde zu „Laborratten“. Diese Diskussion führte zu der Entscheidung des Projektkonsortiums, in der Region nicht mit dem Begriff „Reallabor“ zu operieren, sondern unter dem Titel „Wissensdialog Nordschwarzwald" zu arbeiten.

Diese Episode lässt uns abschließend die vorsichtige Vermutung anstellen, dass die Reallaborforschung mit ihrem spezifischen Jargon der „Transition bzw. City Labs“ im Moment noch stark auf urbane Milieus zugeschnitten ist. Angesichts der Bedeutung ländlicher Räume für die gesellschaftliche Nachhaltigkeitstransformation und der im Projekt WiNo bereits gewonnenen, oben geschilderten Erfahrungen gilt es nun, eine auf den ländlichen Raum angepasste Reallaborforschung zu entwickeln. Wie diese ausgestaltet sein könnte, darauf hoffen wir mit Abschluss der zweiten Projekthälfte detailliertere Auskünfte geben zu können.

\section{Danksagung}

Wir danken: dem Ministerium für Wissenschaft, Forschung und Kunst (MWK) Baden-Württemberg für die Projektförderung, der Begleitforschung der Reallabore, den WiNo-Forschungsinstitutionen und PraxispartnerInnen, allen in WiNo mitwirkenden Kolleginnen und Kollegen sowie Laura Schmid für die Durchführung der Interviews. 


\section{Literatur}

Bauer, A.; Pregernig, M., 2013: Whose Look Into the Future? Participation in Technology Assessment and foresight. In: Critical Policy Studies 7/1 (2013), S. $18-36$

Bauer, A.; Pregernig, M.; Reinecke, S., 2016: Enacting Effective Climate Policy Advice: Institutional Strategies to Foster Saliency, Credibility and Legitimacy. In: Evidence \& Policy: A Journal of Research, Debate and Practice 12/3 (2016), S. 341-362

Bergmann, M.; Jahn, T., 2005: Qualitätskriterien transdisziplinärer Forschung. Ein Leitfaden für die formative Evaluation von Forschungsprojekten. Frankfurt a. M.

Bruckmeier, K.; Tovey, H., 2009: Rural Sustainable Development in the Knowledge Society. Farnham

Eser, U.; Benzing, B.; Müller, A., 2013: Gerechtigkeitsfragen im Naturschutz: Was sie bedeuten und warum sie wichtig sind. Münster

Friedel, R.; Spindler, E.A., 2009: Nachhaltige Entwicklung ländlicher Räume. Chancenverbesserung durch Innovation und Traditionspflege. Wiesbaden

Future Earth, 2013: Future Earth Initial Design: Report of the Transition Team. Paris

Hegger, D.; Lamers, M.; van Zeijl-Rozema, A. et al., 2012: Conceptualising joint knowledge production in regional climate change adaptation projects: success conditions and levers for action. In: Environmental Science \& Policy 18 (2012), S. 52-65

Hubo, C.; Krott, M., 2015: Macht von Politiksektoren als Chance für Wandel am Beispiel Waldnaturschutz. In: Partzsch, L.; Weiland, S. (Hg.): Macht und Wandel in der Umweltpolitik: ZPol Sonderband 2015 II. Baden-Baden, S. 29-56

Hurni, H.; Wiesmann, U., 2004: Towards Transdisciplinarity in Sustainability-oriented Research for Development. In: Hurni, H.; Wiesmann, U.; Schertenleib, R. (Hg.): Research for Mitigating Syndromes of Global Change. A Transdisciplinary Appraisal of Selected Regions of the World to Prepare Development-Oriented Research Partnerships, S. 31-41

Jahn, T.; Bergmann, M.; Keil, F., 2012: Transdisciplinarity. In: Ecological Economics: The Transdisciplinary Journal of the International Society for Ecological Economics 79 (2012), S. 1-10

Jasanoff, S., 2004: States of Knowledge. The Co-production of Science and Social Order. London

Marsden, T., 2009: Mobilities, Vulnerabilities and Sustainabilities: Exploring Pathways from Denial to Sustainable Rural Development. In: Sociologia Ruralis 49/2 (2009), S. 113-131
Matthiesen, U.; Bürkner, H.-J., 2004: Wissensmilieus - Zur sozialen Konstruktion und analytischen Rekonstruktion eines neuen Sozialraum-Typus. In: Matthiesen, U. (Hg.): Stadtregion und Wissen: Analysen und Plädoyers für eine wissensbasierte Stadtpolitik. Wiesbaden, S. 65-89

Nevens, F.; Frantzeskaki, N.; Gorissen, L. et al., 2013: Urban Transition Labs: Co-creating Transformative Action for Sustainable Cities. In: Special Issue: Advancing Sustainable Urban Transformation 50 (2013), S. $111-122$

Penker, M.; Muhar, A., 2015: What's Actually New About Transdisciplinarity? How Scholars from Applied Studies Can Benefit from Cross-disciplinary Learning Processes on Transdisciplinarity. In: Gibbs, S. (Hg.): Transdisciplinary Professional Learning and Practice. Cham, S. 135-147

Pohl, C.; Rist, S.; Zimmermann, A. et al., 2010: Researchers' Roles in Knowledge Co-production: Experience from Sustainability Research in Kenya, Switzerland, Bolivia and Nepal. In: Science and Public Policy 37/4 (2010), S. 267-281

Polk, M., 2015: Transdisciplinary Co-production: Designing and Testing a Transdisciplinary Research Framework for Societal Problem Solving. In: „Advances in Transdisciplinarity 2004-2014““ 65 (2015), S. $110-122$

Reimer, B.; Brett, M., 2013: Scientific Knowledge and Rural Policy: A Long-distant Relationship. In: Sociologia Ruralis 53/3 (2013), S. 272-290

Shortall, S., 2013: Sociology, Knowledge and Evidence in Rural Policy Making. In: Sociologia Ruralis 53/3 (2013), S. 265-271

SRU - Rat von Sachverständigen für Umweltfragen, 1996: Konzepte einer dauerhaft-umweltgerechten Nutzung ländlicher Räume. Sondergutachten Februar 1996. Stuttgart

van Kerkhoff, L., 2014: Developing Integrative Research for Sustainability Science Through a Complexity Principles-based Approach. In: Sustainability Science 9/2 (2014), S. 143-155

\section{Kontakt}

Dr. Regina Rhodius

Albert-Ludwigs-Universität Freiburg

Professur für Fernerkundung und

Landschaftsinformationssysteme (FeLis)

Tennenbacher Straße 4, 79106 Freiburg i.Br.

Tel.: +49 761 - 2033701

E-Mail: regina.rhodius@felis.uni-freiburg.de

《〉 